\title{
Positron emission tomography measurement of brain MAO-B inhibition in patients with Alzheimer's disease and elderly controls after oral administration of sembragiline
}

\author{
Stefan Sturm ${ }^{1,8} \cdot$ Anton Forsberg $^{2} \cdot$ Stephane Nave $^{1} \cdot$ Per Stenkrona $^{2}$. \\ Nicholas Seneca ${ }^{3} \cdot$ Andrea Varrone $^{2} \cdot$ Robert A. Comley ${ }^{7}$ Patrik Fazio $^{2}$. \\ Candice Jamois $^{1} \cdot$ Ryuji Nakao $^{2}$ - Zbigniew Ejduk ${ }^{4}$. Nabil Al-Tawil ${ }^{5}$ - Ulrika Akenine ${ }^{6}$. \\ Christer Halldin $^{2} \cdot$ Niels Andreasen ${ }^{6} \cdot$ Benedicte Ricci $^{1}$
}

Received: 1 April 2016/Accepted: 29 August 2016 / Published online: 16 September 2016

(C) The Author(s) 2016. This article is published with open access at Springerlink.com

\begin{abstract}
Purpose In Alzheimer's disease (AD), increased metabolism of monoamines by monoamine oxidase type B (MAO-B) leads to the production of toxic reactive oxygen species (ROS), which are thought to contribute to disease pathogenesis. Inhibition of the MAO-B enzyme may restore brain levels of monoaminergic neurotransmitters, reduce the formation of toxic ROS and reduce neuroinflammation (reactive astrocytosis), potentially leading to neuroprotection. Sembragiline (also referred as RO4602522, RG1577 and EVT 302 in previous communications) is a potent, selective and reversible inhibitor of MAO-B developed as a potential treatment for AD.

Methods This study assessed the relationship between plasma concentration of sembragiline and brain MAO-B inhibition in patients with $\mathrm{AD}$ and in healthy elderly control (EC) subjects. Positron emission tomography (PET) scans using $\left[{ }^{11} \mathrm{C}\right]_{-}$-deprenyl- $\mathrm{D}_{2}$ radiotracer were performed in ten patients with $\mathrm{AD}$
\end{abstract}

Stefan Sturm and Anton Forsberg contributed equally to this work.

Electronic supplementary material The online version of this article (doi:10.1007/s00259-016-3510-6) contains supplementary material, which is available to authorized users.

Stefan Sturm

stefan.sturm@roche.com

1 Roche Innovation Center Basel, Roche Pharmaceutical Research and Early Development, Grenzacherstrasse 124, Basel, Switzerland

2 Department of Clinical Neuroscience, Centre for Psychiatric Research, Karolinska Institutet, Stockholm, Sweden

3 AstraZeneca Translational Science Center, Stockholm, Sweden

4 Internal Disease and Gastroenterology, Miedzyleski Specialistic Hospital, Warsaw, Poland and six EC subjects, who received sembragiline each day for 6-15 days.

Results At steady state, the relationship between sembragiline plasma concentration and MAO-B inhibition resulted in an $\mathrm{E}_{\max }$ of $\sim 80-90 \%$ across brain regions of interest and in an $\mathrm{EC}_{50}$ of $1-2 \mathrm{ng} / \mathrm{mL}$. Data in patients with $\mathrm{AD}$ and EC subjects showed that near-maximal inhibition of brain MAO-B was achieved with $1 \mathrm{mg}$ sembragiline daily, regardless of the population, whereas lower doses resulted in lower and variable brain MAO-B inhibition.

Conclusions This PET study confirmed that daily treatment of at least $1 \mathrm{mg}$ sembragiline resulted in near-maximal inhibition of brain MAO-B enzyme in patients with AD.

Keywords Monoamine oxidase inhibitor $\cdot$ MAO-B · Sembragiline $\cdot$ Alzheimer's disease $\cdot$ Positron emission tomography

Karolinska Trial Alliance Phase 1 Unit, Karolinska University Hospital, Stockholm, Sweden

6 Karolinska Institutet Alzheimer Disease Research Centre and Clinical Trial Unit, Geriatric Clinic, Karolinska University Hospital, Huddinge, Sweden

AbbVie, North Chicago, IL, USA

8 F. Hoffmann-La Roche Ltd, Grenzacherstrasse 124, 4070 Basel, Switzerland 


\section{Introduction}

Expression of brain monoamine oxidase type B (MAO-B) increases with age in healthy subjects [1,2], and is further increased in patients with Alzheimer's disease (AD) [3, 4]. The brain areas with the greatest increase of MAO-B in patients with $\mathrm{AD}$, ranging from 40 to $60 \%$, are also known to contain high densities of senile plaques and tangles (temporal and parietal cortices and hippocampus) [5]. Moreover, immunohistochemical and autoradiographic studies in AD postmortem brain tissue have shown that MAO-B is highly expressed in reactive astrocytes (gliosis) surrounding amyloid plaques [6].

This up-regulation of MAO-B appears from an early stage, and the increased activity is maintained throughout the course of the disease [7], suggesting that MAO-B may contribute to neurodegenerative processes by increasing oxidative stress in at least two ways. Deamination of dopamine and other amines by MAO-B leads to the formation of hydrogen peroxide that, in the presence of trace amounts of metals, can be converted into highly toxic hydroxyl radicals. Such radicals react avidly with polyunsaturated fats in neuronal membranes, initiating lipid peroxidation and cell death [8]. Secondly, increases in MAO-B may also contribute to the reduced neurotransmitter levels in the brains of $\mathrm{AD}$ patients which may lead to the neuropsychological symptoms that are common in AD [6].

In humans, MAO-B preferentially deaminates dopamine and phenylethylamine and has been suggested to participate in the catabolism of noradrenaline [8]. In addition, MAO-B is highly expressed in serotonin-containing neurons such as those in the raphe nucleus. Dopamine and serotonin [9] levels are reported to be decreased in the brains of individuals with AD [10]. Thus, inhibition of MAO-B enzymatic activity is expected to produce beneficial effects in $\mathrm{AD}$ by a dual mechanism, namely, by reducing neurodegenerative processes due to oxidative stress and by re-establishing a physiological tonus of monoaminergic neurotransmission.

Additional evidence for the efficacy of MAO-B inhibitors (MAO-Bis) in the treatment of AD comes from clinical trials with the MAO-Bi selegiline. The results of a 2-year trial in patients with moderate $\mathrm{AD}$ suggest that selegiline may delay functional deterioration and disease progression [11]. In addition, lazabemide, a selective MAO-Bi developed by Roche but not continued due to hepatotoxic liability, showed a significant improvement in cognition and functionality in patients with $\mathrm{AD}$ at doses previously shown in a positron emission tomography (PET) study with healthy volunteers to provide full blockade of the enzyme [12].

Sembragiline is a potent, selective and slowly reversible MAO-Bi that has a novel chemical structure different from that of lazabemide and selegiline. Based on MAO enzymatic activity and displacement assays, the observed $\mathrm{IC}_{50}$ value from in vitro affinity assays of the MAO-B enzyme is 5-6
$\mathrm{nM}$ [13]. This is approximately 600 -fold more potent in inhibiting MAO-B than MAO-A, and can thus achieve complete MAO-B inhibition without affecting the activity of MAO-A, as well as a wide variety of receptors, ion channels or other enzymes.

Approximately 1040 subjects have participated in phase 1 and 2 sembragiline studies for smoking cessation and moderate $\mathrm{AD}$ dementia [14]. In these studies, sembragiline was found to be safe and well tolerated. At low doses, the pharmacokinetics (PK) of sembragiline are less than dose-proportional, but after administration of approximately $5 \mathrm{mg}$ of sembragiline, the PK become linear, dose-proportional and time-invariant, due to saturation of capacity-limited binding to the peripheral enzyme receptor. In steady-state conditions, sembragiline is slowly eliminated from the plasma, with a terminal half-life of about $50-65 \mathrm{~h}$ in elderly subjects.

PET imaging studies in healthy young men confirmed that sembragiline crosses the blood-brain barrier and inhibits brain MAO-B enzyme activity. A dose-related increase in brain MAO-B inhibition was demonstrated in young healthy subjects who received single doses of 5 and $15 \mathrm{mg}$ sembragiline. Doses of $5 \mathrm{mg}$ sembragiline, administered daily for $2-10$ days, resulted in 52-78 \% and 73-87\% inhibition in cortical and subcortical regions, respectively (Roche data on file). At a dose of $15 \mathrm{mg}$ sembragiline, 68-74\% and 83-86\% inhibition of brain MAO-B was observed in cortical and subcortical regions, respectively; selegiline $20 \mathrm{mg}$, which was used as active comparator in the study, provided mean inhibition levels of 46-79\% for the different brain regions. Based on simulations with a population PK-enzyme inhibition model developed with data from 36 young healthy volunteers, a daily dose of $1 \mathrm{mg}$ sembragiline is anticipated to reach near full MAO-B inhibition in most patients with $\mathrm{AD}$, assuming no relevant age or disease effect on the PK-MAO-B inhibition relationship (Roche data on file).

The present repeated-dose PET study was undertaken to confirm near-complete MAO-B enzyme inhibition in the brain at the doses tested in a phase 2 clinical trial in patients with moderate $A D$, registered at ClinicalTrials.gov (NCT01677754). The objectives of this PET study were to determine the effects of varying multiple oral doses of sembragiline at steady state on brain MAO-B enzyme activity in patients with mild-to-moderate $\mathrm{AD}$ using $\mathrm{PET}$ imaging with $\left[{ }^{11} \mathrm{C}\right]{ }_{\mathrm{L}}$-deprenyl- $\mathrm{D}_{2}$, and to characterise the sembragiline plasma-brain MAO-B inhibition relationship in patients with $\mathrm{AD}$ compared to healthy age-matched controls.

\section{Material and methods}

The study was approved by the Regional Ethical Review Board in Stockholm, Sweden, the Radiation Safety Committee at the Karolinska University Hospital in Solna 
(Stockholm, Sweden) and the Medical Product Agency in Sweden. Patients with AD and healthy elderly controls (EC) were enroled in Karolinska Hospital at Huddinge, Sweden.

\section{Study design}

In this multicentre, open-label, parallel-group, multiple-dose study, sembragiline was administered orally once daily for 615 days, depending on the dose group, to reach PK and enzyme inhibition steady-state conditions, and to allow for flexibility in PET scan scheduling. The first dose was administered at the study centre under the supervision of study personnel. On subsequent study days, treatment compliance was assured by intake logs and tablet counting.

At baseline, each subject underwent a PET scan using $\left[{ }^{11} \mathrm{C}\right]-\mathrm{L}$-deprenyl- $\mathrm{D}_{2}$ to measure MAO-B enzyme activity in the brain prior to their first dose of allocated medication. The second scan was performed at trough concentrations at steady state prior to the last drug administration. Before each scan, a cannula was inserted into a radial artery and another into an antecubital vein. A sterile phosphate buffer $(\mathrm{pH}=7.4)$ containing radioligand was injected as a bolus over several seconds into the cubital vein.

\section{Subjects: inclusion, exclusion criteria}

Healthy EC subjects with no evidence of AD or other cognitive impairment and patients with $\mathrm{AD}$ aged $50-80$ years were enroled in the study. Informed consent was obtained from all EC subjects and patients with $\mathrm{AD}$, co-signed by the patient's closest relative and, for patients who were incapable of giving informed consent, a legally authorised representative.

Patients with probable AD based on the National Institute of Neurological and Communicative Disorders and Stroke and the Alzheimer's Disease and Related Disorders Association (NINCDS/ADRDA) and the Diagnostic and Statistical Manual of Mental Disorders, 4th Edition (DSMIV) criteria, having a Mini-Mental State Exam (MMSE) score of 17-26 inclusive, and a modified Hachinski Ischemia Scale score $\leq 4$ were enroled in the study. A neuroimaging evaluation of the brain by magnetic resonance imaging (MRI) supported the diagnosis of $\mathrm{AD}$, with no evidence of focal disease to account for dementia or MRI exclusion criteria.

Background AD therapy of anticholinesterase inhibitors (donepezil, galantamine, rivastigmine), antidepressants (citalopram, paroxetine, sertraline), antipsychotics (risperidone, quetiapine), and memantine were permitted, provided that they were maintained on a stable dosage regimen for at least 1 month before day 1 of the study. Further benzodiazepines with a short half-life (e.g. lorazepam), zolpidem, zopiclone, eszopiclone and trazodone for insomnia were permitted in patients with AD. Occasional use of oxazepam or lorazepam for anxiety due to MRI scan was permitted in all subjects.

EC subjects showed no clinically relevant findings on physical examination, vital signs, electrocardiography (ECG) or routine laboratory tests, and no suspicion of cognitive impairment or early dementia on the Rey Auditory Verbal Learning Test, MMSE, copying a cube and cross shown on paper, and drawing a time point on a clock. EC subjects were excluded if they had a family history of AD in first- or seconddegree relatives $<75$ years of age. All subjects were instructed to follow a low-tyramine diet.

Exclusion criteria for patients with $\mathrm{AD}$ and $\mathrm{EC}$ subjects included prior exposure to ionising radiation or radioisotope for research that would exceed the local yearly radiation dose exposure, and implants or ferromagnetic foreign bodies that would present a risk during MRI.

\section{Study treatment}

For dosing with target doses $<1 \mathrm{mg}$, a loading dose of $5 \mathrm{mg}$ was administered on day 1 to saturate the capacity-limited binding of sembragiline, allowing achievement of steadystate levels (PK and pharmacodynamic [PD]) within 7-14 days of dosing.

Group 1 was composed of three patients with $\mathrm{AD}$ and three EC subjects who received $1 \mathrm{mg}$ sembragiline daily for 2 weeks. This dose regimen was expected to provide enzyme inhibition levels in the range of $80 \%$ averaged across brain regions of interest (ROIs). A loading dose of $5 \mathrm{mg}$ was to be administered on day 1 of treatment, followed by daily administration of target doses for doses $<1 \mathrm{mg}$.

Group 2 was composed of three patients with $\mathrm{AD}$ and three EC subjects who received a loading dose of $5 \mathrm{mg}$ on day 1 and doses of $0.2 \mathrm{mg} /$ day for the following 6 days. This dose regimen was expected to provide enzyme inhibition levels in the range of 60-70\% averaged across brain ROIs.

Group 3 was composed of two patients with AD who received a dose of $5 \mathrm{mg}$ of sembragiline in order to better characterise the plateau of enzyme inhibition in patients with AD.

Group 4 was composed of two patients with AD who received a 5-mg loading dose on day 1 , followed by $0.1 \mathrm{mg}$ of sembragiline for 13-16 days, to explore enzyme inhibition in lower concentration ranges. Male subjects were selected for group 4 in order to reduce the gender imbalance between the patients with $\mathrm{AD}$ and the $\mathrm{EC}$ subjects.

\section{Safety assessments}

The safety of sembragiline was assessed by monitoring vital signs, laboratory tests and adverse events (AEs), and by 
evaluating the risk of suicide using the Columbia Suicide Severity Rating Scale.

\section{Synthesis/preparation of $\left[{ }^{11} \mathrm{C}\right]_{-}-{ }_{-}$deprenyl-D ${ }_{2}$}

$\left[{ }^{11} \mathrm{C}\right]-_{\mathrm{L}}$-deprenyl- $\mathrm{D}_{2}$ was prepared by $N$-methylation of the desmethyl ${ }_{L}$-deprenyl- $\mathrm{D}_{2}$ precursor using $\left[{ }^{11} \mathrm{C}\right]$ methyl triflate at room temperature [15]. The radiochemical yield was 40 $50 \%$, and the radiochemical purity was $>95 \%$. The entire synthesis was performed using a completely automated commercial radiochemistry module (Scansys PET chemistry module; Scansys Laboratorieteknik, Denmark).

\section{MRI}

All subjects underwent MRI, performed on a 1.5-T Siemens MAGNETOM Avanto scanner at Medicinsk Röntgen, Odenplan, Stockholm, Sweden. T1-weighted MR images were obtained for each individual and used for grey and white matter segmentation and delineation of ROIs using the Anatomical Automatic Labeling (AAL) template [16].

The MR image was segmented into grey and white matter, and cerebrospinal fluid segments, using the SPM5 segmentation algorithm in MATLAB (Wellcome Trust Centre for Neuroimaging, London, UK; The MathWorks, Inc., Natick, MA, USA). ROIs encompassing the lateral frontal cortex, medial frontal cortex, lateral temporal cortex, and lateral parietal cortex, lateral occipital cortex, hippocampus, putamen, thalamus, cerebellum and whole brain were drawn on the co-registered MR image and mapped onto the PET images, and time-activity curves were extracted.

\section{PET scan procedures}

A transmission scan was performed using three rotating ${ }^{68} \mathrm{Ge}$ rod sources. $\left[{ }^{11} \mathrm{C}\right]-\mathrm{L}-$-Deprenyl- $\mathrm{D}_{2}$ was injected intravenously as a bolus, and emission data were acquired over $63 \mathrm{~min}$ using the ECAT EXACT high-resolution system (Siemens Medical Solutions, Malvern, PA, USA). The injected radioactivity was targeted to approximately $300 \mathrm{MBq}$. The injected mass of deprenyl was to be $\leq 100 \mu \mathrm{g}$, and $\geq 50-100$-fold lower than the typical administered dose of $5-10 \mathrm{mg}$ deprenyl to patients with Parkinson's disease. The framing was as follows: 9 frames x $10 \mathrm{~s}, 2$ frames x $15 \mathrm{~s}, 3$ frames x $20 \mathrm{~s}, 4$ frames x $30 \mathrm{~s}, 4$ frames x $60 \mathrm{~s}, 4$ frames x $180 \mathrm{~s}, 7$ frames x $360 \mathrm{~s}$. Images were reconstructed using the standard filtered backprojection, with a 2-mm Hanning filter, a zoom factor of 2.17, and a $128 \times 128$ matrix, and were corrected for attenuation and scatter [17]. A summation PET image was created for each subject and PET session by averaging across all frames. Time-activity curves were then extracted from selected ROIs.

\section{Arterial blood sampling}

Arterial blood samples were used for measurement of radioactivity in whole blood and plasma, as well as to determine the parent fraction of the radioligand, using a reversed-phase high-performance liquid chromatography method (Karolinska University Hospital, Solna, Stockholm, Sweden). A catheter was inserted in the radial artery, and arterial blood was collected continuously for $10 \mathrm{~min}$ using an automated blood sampling system at a speed of $5 \mathrm{~mL} /$ min. Discrete blood samples were drawn approximately 10 , $15,20,30,45$ and 60 min after each injection of $\left[{ }^{11} \mathrm{C}\right]_{-} \mathrm{L}^{-}$ deprenyl- $\mathrm{D}_{2}$.

\section{Quantitation of $\left[{ }^{11} \mathrm{C}\right] \mathrm{-}_{\mathrm{L}}$-deprenyl- $\mathrm{D}_{2}$ binding}

Time-activity data were analysed as previously described [18, 19]. Briefly, $\left[{ }^{11} \mathrm{C}\right]_{-\mathrm{L}}$-deprenyl- $\mathrm{D}_{2}$ was quantified by the twotissue compartment model with three rate constants $\left(K_{1}, k_{2}, k_{3}\right)$ using the PMOD 3.3 software package (PMOD Group, Zurich, Switzerland), where the tissue compartments represent the free plus non-specifically bound and specifically bound radiotracer. The outcome measure $\lambda k_{3}$ was calculated as $\left(K_{1} / k_{2}\right) * k_{3}$, using a non-linear approach, and was used as an estimate for specific binding to MAO-B that was independent of perfusion. The parameter $\lambda \mathrm{k} 3$ (where $\lambda=\mathrm{K} 1 / \mathrm{k} 2$ ) was used as an estimate for specific binding to MAO-B, as it was found to reflect the regional enzyme concentration more accurately than $\mathrm{k} 3$ alone [19]. This is because $\lambda \mathrm{k} 3$ is independent of perfusion and has better reproducibility than $\mathrm{k} 3$ alone [20].

Inhibition of MAO-B in the brain by sembragiline from regional $\lambda k_{3}$ was calculated as follows:

Inhibition $=100 \% x\left(\mid \lambda k_{3}\right.$ drug $-\lambda k_{3}$ baseline $\mid / \lambda k_{3}$ baseline $)$

Here, "drug" refers to scans after drug administration.

\section{Assessment of PK}

Blood samples were collected for sembragiline plasma concentrations and analysed with a validated liquid chromatography-tandem mass spectrometry method (Covance Inc., Harrogate, North Yorkshire, UK). Plasma concentrations of sembragiline were measured just prior to the second PET scan. 


\section{Sembragiline plasma concentration-MAO-B enzyme inhibition relationship}

Pre-scan plasma concentrations were used for the analysis of the relationship between MAO-B enzyme inhibition and steady-state sembragiline plasma concentration, and were characterised using a simple $\mathrm{E}_{\max }$ model for each brain ROI:

$\operatorname{Occ}(\%)=E_{\max } * C_{p} /\left(C_{p}+E C_{50}\right)$

, where $\mathrm{C}_{\mathrm{p}}=0 \mathrm{ng} / \mathrm{mL}$ at baseline and $\mathrm{C}_{\mathrm{p}}=$ infinity $(\mathrm{ng} / \mathrm{mL})$ at $\mathrm{E}_{\max }$; and where $\mathrm{C}_{\mathrm{p}}$ is sembragiline plasma concentration (ng/ $\mathrm{mL}), \mathrm{E}_{\max }$ is maximum MAO-B enzyme inhibition (\%) and $\mathrm{EC}_{50}$ is sembragiline concentration $(\mathrm{ng} / \mathrm{mL})$ at half-maximal inhibition.

The PK/PD analysis was performed using Phoenix WinNonlin software version 6.2 (Pharsight Corporation, Mountain View, CA, USA).

\section{Results}

Seventeen subjects (11 patients with $\mathrm{AD}$ and six EC subjects) were enroled in the study. One enroled subject, a patient with $\mathrm{AD}$ assigned to group 1, withdrew from the study because it was not possible to perform arterial cannulation, and thus no baseline PET scan was performed and no study treatment was administered.

The demographics of the 16 patients who received sembragiline are summarised in Supplementary Table 1. Patients with $\mathrm{AD}$ were on stable background $\mathrm{AD}$ therapy with galantamine, donepezil, memantine, or a combination of galantamine and memantine. All subjects were non-smokers. There was an imbalance in gender between the patients with $\mathrm{AD}$ and the healthy EC subjects. The majority $(80 \%)$ of patients with $\mathrm{AD}$ were female, whereas the majority $(83 \%)$ of EC subjects were male. Healthy control subjects (70.5 years) were on average slightly older than the $\mathrm{AD}$ patients (65.0 years).

Table 1 Regional $\left[{ }^{11} \mathrm{C}\right]_{-}$-deprenyl- $\mathrm{D}_{2} \lambda \mathrm{k} 3$ values before and after sembragiline treatment and respective occupancy values induced by the drug regimens by population

\begin{tabular}{|c|c|c|c|c|c|c|c|}
\hline & & Whole brain & Hippocampus & Striatum & Thalamus & Frontal cortex & Parietal cortex \\
\hline $\mathrm{AD}$ & Baseline & $0.18(0.16-0.21)$ & $0.24(0.20-0.27)$ & $0.30(0.27-0.33)$ & $0.28(0.21-0.34)$ & $0.17(0.14-0.19)$ & $0.17(0.15-0.19)$ \\
\hline $0.1 \mathrm{mg}$ & Post-dose & $0.08(0.08-0.09)$ & $0.11(0.10-0.11)$ & $0.12(0.11-0.13)$ & $0.11(0.09-0.1)$ & $0.07(0.06-0.08)$ & $0.07(0.07-0.08)$ \\
\hline $\mathrm{N}=2$ & Occ, $\%$ & $55.2(52.5-57.9)$ & $53.8(49.2-58.4)$ & $59.9(58.3-61.6)$ & $60.4(56.7-64.1)$ & $56.9(54.8-59.0)$ & $55.6(53.4-57.7)$ \\
\hline $\mathrm{EC}$ & Baseline & $0.16(0.15-0.16)$ & $0.23(0.22-0.24)$ & $0.27(0.26-0.28)$ & $0.25(0.24-0.27)$ & $0.15(0.15-0.16)$ & $0.15(0.14-0.16)$ \\
\hline $0.2 \mathrm{mg}$ & Post-dose & $0.08(0.07-0.10)$ & $0.11(0.09-0.13)$ & $0.12(0.09-0.14)$ & $0.12(0.09-0.13)$ & $0.07(0.06-0.09)$ & $0.07(0.06-0.09)$ \\
\hline$N=3$ & Occ, $\%$ & $49.1(37.5-58.8)$ & $51.6(42.3-60.9)$ & $56.1(47.9-66.3)$ & $53.7(44.5-66.6)$ & $51.7(41.9-63.5)$ & $50.7(37.2-62.3)$ \\
\hline $\mathrm{AD}$ & Baseline & $0.20(0.18-0.24)$ & $0.31(0.26-0.36)$ & $0.35(0.29-0.45)$ & $0.31(0.26-0.36)$ & $0.21(0.18-0.26)$ & $0.20(0.17-0.25)$ \\
\hline $0.2 \mathrm{mg}$ & Post-dose & $0.06(0.06-0.07)$ & $0.07(0.07-0.08)$ & $0.08(0.08-0.08)$ & $0.07(0.07-0.07)$ & $0.06(0.05-0.07)$ & $0.06(0.05-0.07)$ \\
\hline$N=3$ & Occ, $\%$ & $68.9(64-75.2)$ & $76.1(70.7-81.1)$ & $77.1(72.5-83.3)$ & $76.8(71.7-80.6)$ & $70.2(60.4-79.4)$ & $68.7(57.1-79.3)$ \\
\hline $\mathrm{EC}$ & Baseline & $0.19(0.19-0.20)$ & $0.28(0.25-0.31)$ & $0.35(0.33-0.38)$ & $0.32(0.29-0.36)$ & $0.18(0.17-0.19)$ & $0.18(0.15-0.20)$ \\
\hline $1 \mathrm{mg}$ & Post-dose & $0.05(0.04-0.05)$ & $0.04(0.03-0.05)$ & $0.05(0.04-0.06)$ & $0.05(0.04-0.05)$ & $0.04(0.04-0.05)$ & $0.04(0.04-0.05)$ \\
\hline$N=3$ & Occ, $\%$ & $75.4(73.2-79.2)$ & $83.9(79.7-87.6)$ & $84.7(81.3-87.6)$ & $85.7(82.9-88.2)$ & $76.2(72.7-79.3)$ & $76.1(71.3-79.9)$ \\
\hline $\mathrm{AD}$ & Baseline & $0.16(0.14-0.19)$ & $0.22(0.19-0.28)$ & $0.28(0.24-0.33)$ & $0.25(0.20-0.27)$ & $0.16(0.12-0.22)$ & $0.16(0.11-0.22)$ \\
\hline $1 \mathrm{mg}$ & Post-dose & $0.04(0.04-0.05)$ & $0.05(0.05-0.05)$ & $0.04(0.04-0.05)$ & $0.04(0.04-0.04)$ & $0.04(0.03-0.04)$ & $0.04(0.03-0.05)$ \\
\hline $\mathrm{N}=3,2^{\mathrm{a}}$ & Occ, $\%$ & $75.5(74.9-76.2)$ & $79.2(76.3-82.1)$ & $85.0(83.6-86.4)$ & $86.0(85.7-86.3)$ & 79.7 (79.6-79.9) & $78.0(76.7-79.3)$ \\
\hline $\mathrm{AD}$ & Baseline & $0.19(0.18-0.19)$ & $0.24(0.24-0.24)$ & $0.30(0.29-0.31)$ & $0.28(0.21-0.34)$ & $0.18(0.16-0.19)$ & $0.18(0.17-0.19)$ \\
\hline $5 \mathrm{mg}$ & Post-dose & $0.04(0.04-0.05)$ & $0.04(0.03-0.05)$ & $0.05(0.04-0.05)$ & $0.11(0.09-0.12)$ & $0.04(0.04-0.04)$ & $0.04(0.03-0.04)$ \\
\hline $\mathrm{N}=2$ & Occ, $\%$ & $76.3(73.8-78.7)$ & $83.3(80.3-86.3)$ & $84.8(83.3-86.4)$ & $86.3(84.2-88.5)$ & $77.8(75.2-80.4)$ & $78.1(73.5-82.7)$ \\
\hline $\mathrm{AD}$ & Baseline & $0.18(0.14-0.24)$ & $0.26(0.19-0.36)$ & $0.31(0.24-0.45)$ & $0.28(0.20-0.36)$ & $0.18(0.12-0.26)$ & $0.18(0.11-0.25)$ \\
\hline \multicolumn{8}{|l|}{$\mathrm{N}=10$} \\
\hline $\mathrm{EC}$ & Baseline & $0.18(0.15-0.20)$ & $0.25(0.22-0.31)$ & $0.31(0.26-0.48)$ & $0.29(0.24-0.36)$ & $0.17(0.15-0.19)$ & $0.16(0.14-0.20)$ \\
\hline $\mathrm{N}=10$ & & & & & & & \\
\hline
\end{tabular}

Values are means (range)

Abbreviations: AD, patients with Alzheimer's disease; EC, healthy elderly control; $0.1 \mathrm{mg}$, loading dose of $5 \mathrm{mg}$ on day 1 followed by $0.1 \mathrm{mg}$ q.d. for days 2-15; $0.2 \mathrm{mg}$, loading dose of $5 \mathrm{mg}$ on day 1 followed by $0.2 \mathrm{mg}$ q.d. on days $2-7 ; 1 \mathrm{mg}, 1 \mathrm{mg}$ q.d. for 14 days; $5 \mathrm{mg}, 5 \mathrm{mg}$ q.d. for $6-9$ days

${ }^{a} \mathrm{~N}=3$ for baseline $\lambda \mathrm{k} 3$ values and $\mathrm{N}=2$ for post-dose $\lambda \mathrm{k} 3$ and occupancy values 
Fig. 1 Visualization of MAO-B inhibition in the brain following treatment with $1 \mathrm{mg}$ sembragiline once a day for 2 weeks. Axial, coronal and sagittal slices of $\left[{ }^{11} \mathrm{C}\right]_{-} \mathrm{L}$-deprenyl- $\mathrm{D}_{2}$ PET images from a patient with $\mathrm{AD}$ in the 1$\mathrm{mg}$ group are presented before and after sembragiline treatment

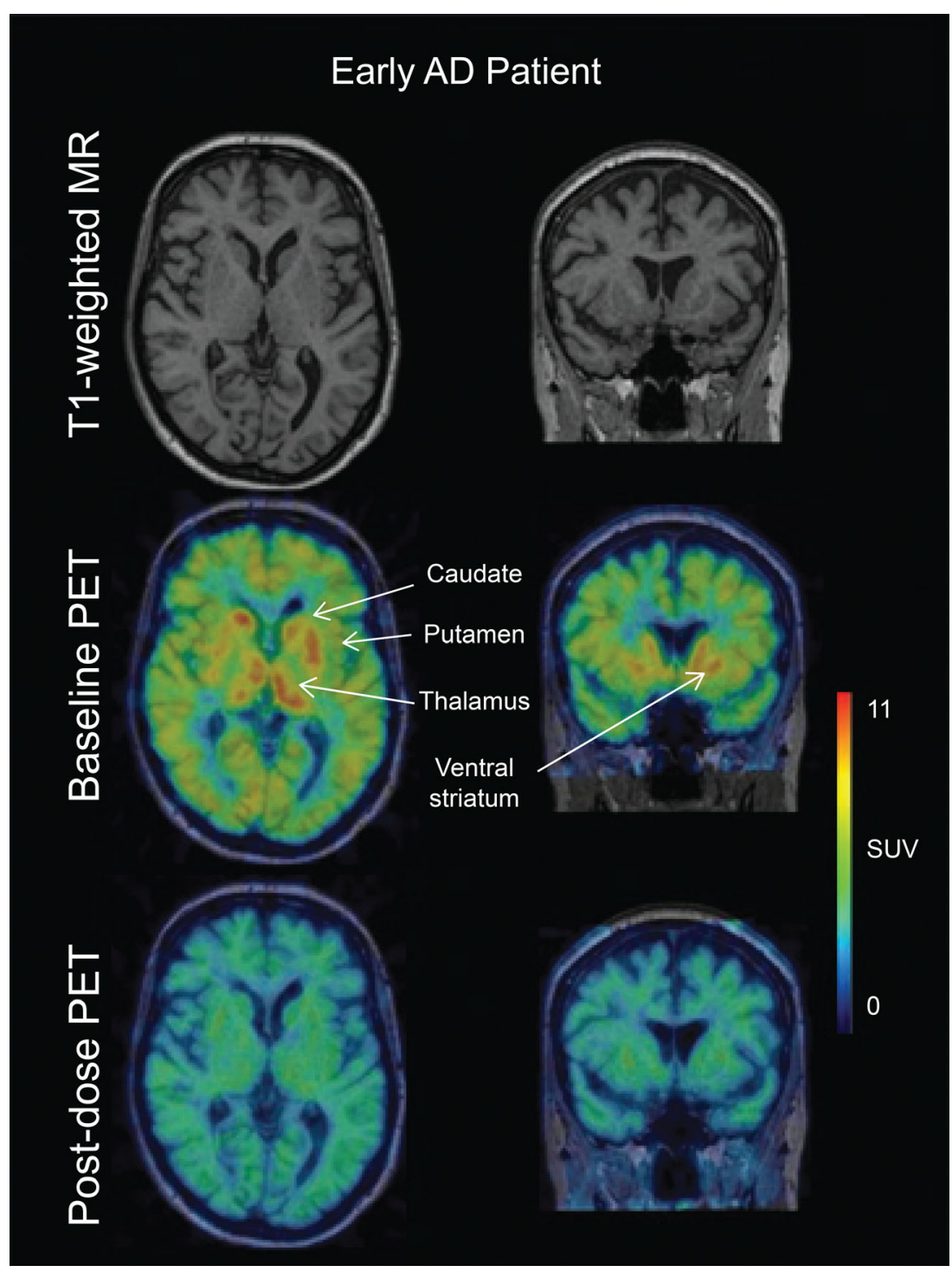

\section{Treatment compliance}

All EC subjects documented daily drug intake and returned the expected number of tablets. Two patients with $\mathrm{AD}$ each missed one dose. Three patients with AD returned fewer tablets than expected at the end of the study.

\section{Safety}

Sembragiline treatment was well tolerated in patients with $\mathrm{AD}$ and EC subjects. No AE led to withdrawal from the study, and all subjects completed their assigned treatment. In total, 11 of 20 AEs were reported as drug-related. Four of six EC subjects experienced 13 AEs during the study, and seven AEs were reported in three of ten patients with AD. Most AEs were of mild intensity. There was no trend for increased AEs with dose and no trend for body system-specific AEs. The most frequently observed $\mathrm{AE}$ was diarrhoea, which occurred in three subjects. There were no clinically significant changes in laboratory parameters, vital signs or ECG measurements.

\section{Sembragiline plasma concentrations}

Mean plasma sembragiline concentrations taken prior to the second PET scan and last dose administration are summarised in Supplementary Table 2. With a loading dose of $5 \mathrm{mg}$ sembragiline for low doses $(<1 \mathrm{mg})$ or multiple doses for higher doses $(\geq 1 \mathrm{mg})$, steady-state conditions were reached within 14 days and at the time of the second PET scan. Plasma concentrations at the time of PET scanning were in the expected range, based on simulations with a populationPK model previously developed in healthy volunteers (Roche data on file). A trend toward higher exposure of sembragiline in patients with $\mathrm{AD}$ ( $80 \%$ female) compared with EC subjects 
was suggested, where $83 \%$ of enroled subjects were male. A gender difference might explain the difference in PK between the two populations.

\section{Dosage of the $\left[{ }^{11} \mathrm{C}\right]-\mathrm{L}$-deprenyl-D2 tracer}

The injected radioactivity and injected mass were $310 \pm$ $35 \mathrm{MBq}$ and $280 \pm 37 \mathrm{MBq}$, and $0.28 \pm 0.16 \mu \mathrm{g}$ and $0.55 \pm$ $0.60 \mu \mathrm{g}$, for $\mathrm{EC}$ subjects and $\mathrm{AD}$ patients, respectively.

A maximum injected mass of $5 \mu \mathrm{g}$ was considered the threshold, and injected doses were well below this value, ensuring there was no effect on inhibition.

Injected radioactivity and total dose of the radiotracer per treatment group, scan and population are listed in Supplementary Table 3.

\section{$\left[{ }^{11} \mathrm{C}\right]-{ }_{\mathrm{L}}-$ Deprenyl- $\mathrm{D}_{2}$ binding in the brain}

$\lambda k_{3}$ data representing specific binding of the $\left[{ }^{11} \mathrm{C}\right]_{-}$-deprenyl$\mathrm{D}_{2}$ tracer to MAO-B enzyme are summarised in Table 1. $\lambda k_{3}$ values before and after treatment are presented. Baseline $\lambda k_{3}$ values in patients with $\mathrm{AD}$ were similar to those observed in healthy EC subjects. In both patients with $\mathrm{AD}$ and $\mathrm{EC}$ subjects, baseline $\lambda k_{3}$ values were highest in the striatum and lower in the cortical regions. Mean regional MAO-B inhibition levels induced by the different drug regimens are shown in Table 1 and visualised in Figs. 1 and 2.

MAO-B inhibition at trough following $1 \mathrm{mg}$ sembragiline treatment was 75-86 \% (mean values) across all brain ROIs and study populations, and was in the same range as that reported with the 5-mg dose in this study (mean values: 76-86 \%). Doses of $0.1 \mathrm{mg}$ and $0.2 \mathrm{mg}$ were associated with lower and highly variable levels of inhibition. Across all treatment groups, the highest levels of inhibition were observed in subcortical regions such as the hippocampus, thalamus and striatum.

Plots of pre-scan sembragiline concentration versus MAO$\mathrm{B}$ inhibition in steady-state conditions showed a sharp increase at low plasma concentrations to a plateau in MAO-B inhibition (Fig. 3). This was observed for both patients with $\mathrm{AD}$ and EC subjects. The plateau occurred at plasma concentrations of $20-50 \mathrm{ng} / \mathrm{mL}$.

At steady state, the sembragiline plasma concentration-inhibition relationship could be described by a simple $\mathrm{E}_{\max }$ model. Estimated $\mathrm{E}_{\max }$ was $80-89 \%$ and $\mathrm{EC}_{50}$ was $1-2 \mathrm{ng} /$ $\mathrm{mL}$ across ROIs and for both populations (Table 2). Variability in PET data estimates was low to moderate, with a coefficient of variation (CV) below $40 \%$ for all parameters. A population-PD model based on data from healthy volunteers and patients with $\mathrm{AD}$ yielded very similar $\mathrm{E}_{\max }$ values, predicting median enzyme inhibition of $>80 \%$ for doses $\geq 1 \mathrm{mg}$ in the hippocampus $24 \mathrm{~h}$ post-last dose (Roche data on file). Slightly lower $\mathrm{EC}_{50}$ values were estimated for patients with $\mathrm{AD}$ than for $\mathrm{EC}$ subjects.

Two patients with $\mathrm{AD}$ received concomitant sedative medication on an as-needed basis; one received $5 \mathrm{mg}$ oxazepam and the other received $5 \mathrm{mg}$ zopiclone. Data from these patients did not reveal any differences compared to other subjects with regard to sembragiline plasma concentrations or MAO-B inhibition. To the best of our knowledge, MAO-B is not a known target of oxazepam or zopiclone.

\section{Discussion}

The purpose of the present imaging study was to determine the relationship between exposure to sembragiline and the
Fig. 2 Regional brain MAO-B inhibition values (\%) associated with the various drug regimens. Error bars represent standard deviations. Abbreviations: AD, patients with Alzheimer's disease; EC, healthy elderly control; MAO-B, monoamine oxidase type B

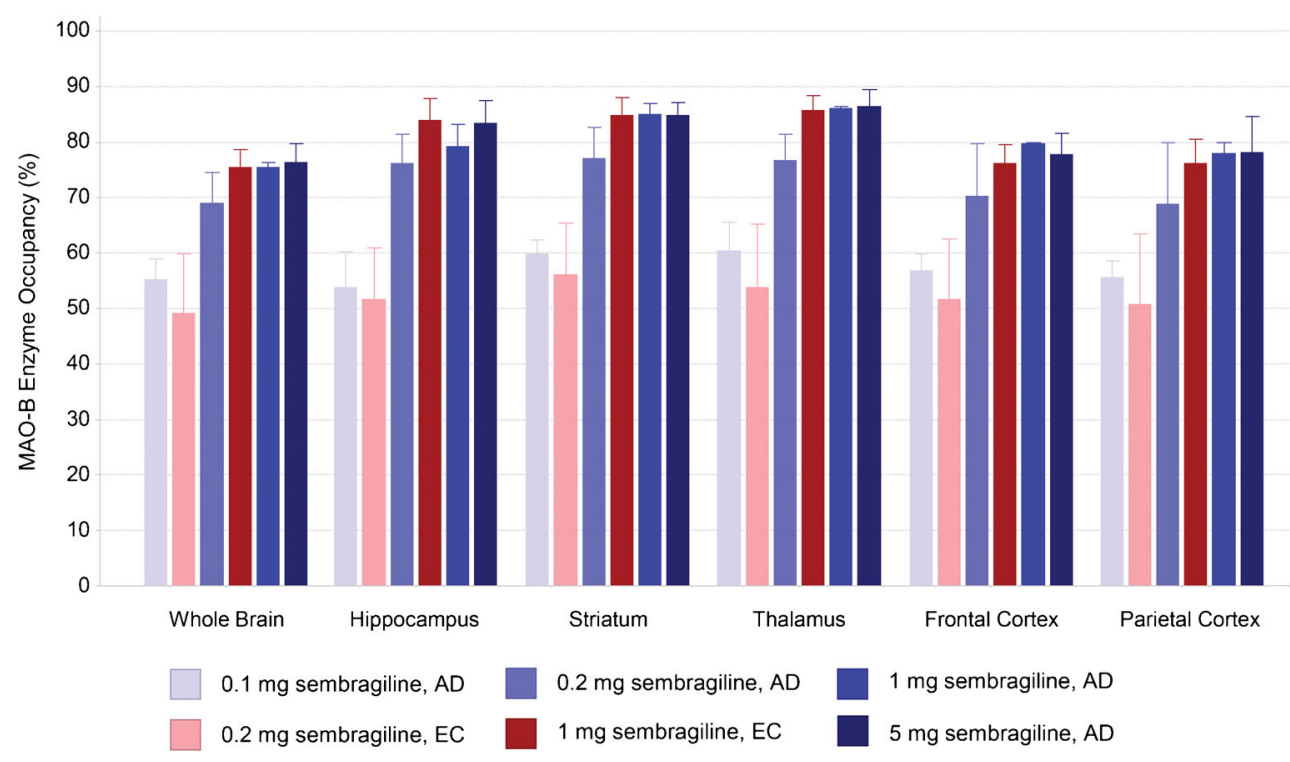



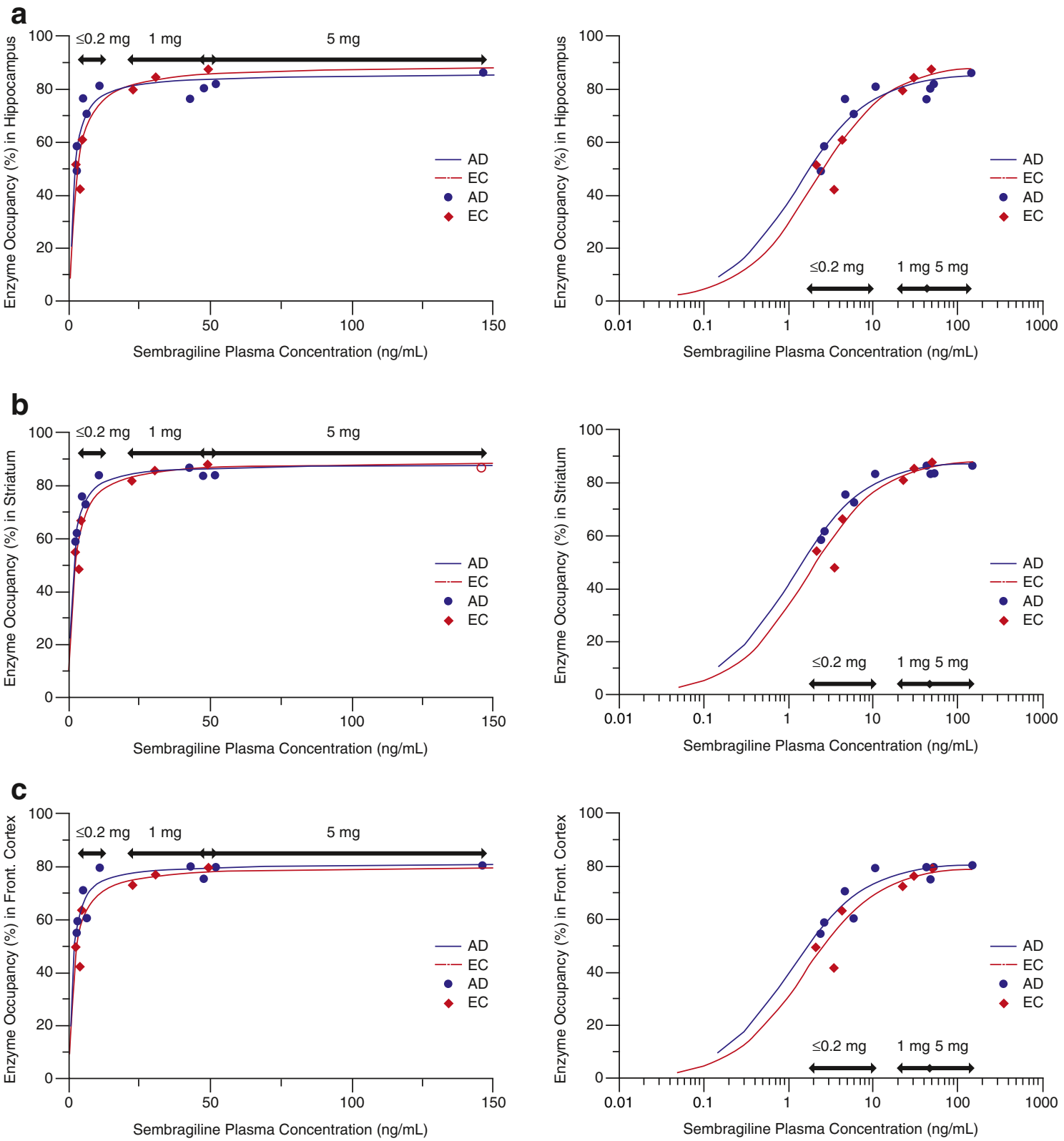

Fig. 3 Relationship between pre-scan plasma concentration of sembragiline and MAO-B inhibition in patients with $\mathrm{AD}$ and EC subjects in steady-state conditions following sembragiline treatment. (a) hippocampus (b) striatum and (c) frontal cortex. Sembragiline was

administered once daily p.o. over 6-15 days at doses of between 0.1 and $5 \mathrm{mg}$. At doses of $0.1 \mathrm{mg}$ and $0.2 \mathrm{mg}$, a loading dose of $5 \mathrm{mg}$ of sembragiline was administered. The arrows represent the range of prescan concentrations at given sembragiline doses

inhibition of MAO-B enzyme activity in the brain after multiple oral doses in patients with AD. These data were used to assist in dose selection of the phase 2 sembragiline study in patients with moderate $\mathrm{AD}$ and in subsequent studies [21].

$\left[{ }^{11} \mathrm{C}\right]-\mathrm{L}$-Deprenyl- $\mathrm{D}_{2}$ was used to determine brain MAO-B inhibition of sembragiline. Previous imaging studies were performed with this radioligand for brain inhibition of MAO-Bis in healthy subjects [22], in elderly subjects in and patients with $\mathrm{AD}$ [23].

MAO-B inhibition following 1-mg treatment of sembragiline was $75-86 \%$ (mean values) across the six brain

ROIs (71-88\% overall) and study populations, and in the same range as those reported for 5-mg doses, indicating that plateau of inhibition had been reached at these doses. Doses of 0.1 and $0.2 \mathrm{mg}$ were associated with lower and highly variable levels of inhibition. At a dose of $0.2 \mathrm{mg}$, women showed higher MAO-B inhibition than men, which is consistent with higher observed concentrations of sembragiline. However, similar MAO-B inhibition between men and women was observed after administration of a 1-mg dose, despite higher sembragiline concentrations in women. This suggests that for near-full MAO-B inhibition doses in the overall 
Table $2 \mathrm{E}_{\max }$ and $\mathrm{EC}_{50}$ values of relationship between pre-scan plasma concentration of sembragiline and MAO-B occupancy of sembragiline in steady-state conditions by population and ROI

\begin{tabular}{llll}
\hline Population & Region of interest & $\mathrm{E}_{\max }, \%$ & $\mathrm{EC}_{50}, \mathrm{ng} / \mathrm{mL}$ \\
\hline EC & Hippocampus & $89.1(\mathrm{CV} \%: 9.6)$ & $2.05(\mathrm{CV} \%: 33.4)$ \\
& Striatum & $88.8(\mathrm{CV} \%: 7.5)$ & $1.67(\mathrm{CV} \%: 29.7)$ \\
& Frontal cortex & $80.1(\mathrm{CV} \%: 8.9)$ & $1.57(\mathrm{CV} \%$ 36.2) \\
$\mathrm{AD}$ & Hippocampus & $85.9(\mathrm{CV} \%: 4.1)$ & $1.29(\mathrm{CV} \%: 21.5)$ \\
& Striatum & $87.8(\mathrm{CV} \%: 1.8)$ & $1.11(\mathrm{CV} \%: 10.7)$ \\
& Frontal cortex & $81.1(\mathrm{CV} \%: 3.2)$ & $1.06(\mathrm{CV} \%: 19.6)$
\end{tabular}

Abbreviations: $\mathrm{CV}$, coefficient of variation; $\mathrm{EC}_{50}$, concentration at halfmaximal occupancy; $\mathrm{E}_{\max }$ maximum MAO-B occupancy; EC, healthy elderly controls, $\mathrm{AD}$, patients with Alzheimer's disease; $\mathrm{ROI}$, region of interest

population, around $1 \mathrm{mg}$ might be needed, and could not be achieved with lower doses $(\leq 0.2 \mathrm{mg})$.

Across all treatment groups, a trend was observed for higher levels of inhibition in subcortical regions such as the hippocampus, thalamus and striatum than in cortical regions. This is consistent with simulated median enzyme inhibition with this compound based on population-PK model findings in healthy young volunteers (Roche data on file), with other MAO-Bi treatment in EC subjects [24], and with patients with $\mathrm{AD}$ [23]. These trends might be explained by non-specific binding of $\left[{ }^{11} \mathrm{C}\right]_{-}-$-deprenyl- $\mathrm{D}_{2}$, resulting in different binding capacity at baseline, or by differences in $K_{1}$, and may be reflective of MAO-B activity, as the binding capacity was highest in the striatum and lower in the cortical regions in both patients with $\mathrm{AD}$ and $\mathrm{EC}$ subjects in the present study as well in others [23, 25].

In this study, baseline $\lambda k_{3}$ values in patients with $\mathrm{AD}$ were similar to those observed in EC subjects, in contrast to previous reports where higher baseline $\left[{ }^{11} \mathrm{C}\right]_{-}-$-deprenyl- $\mathrm{D}_{2}$ binding was observed in patients with $\mathrm{AD}[3,23]$. However, the present analysis is limited by the overall small sample size and the slightly older EC population. When corrected for age, a slight trend for higher $\lambda k_{3}$ in patients with $\mathrm{AD}$ was observed. MAO-B is reduced and inhibited in smokers [26]; however, as all subjects in this study were non-smokers, smoking status was not a confounding factor in the comparison of the two populations.

\section{Concentration-enzyme inhibition relationship}

The mean steady-state inhibition values were in the expected range based on simulations with a preliminary PK enzyme inhibition model. Plots of MAO-B inhibition versus concentration showed a sharp increase at low concentrations, to a plateau in MAO-B inhibition. The relationship between plasma concentration and MAO-B inhibition could be well described with an $\mathrm{E}_{\max }$ model. The estimated $\mathrm{EC}_{50}$ of 1-2 ng/
$\mathrm{mL}$ was comparable to the observed $\mathrm{IC}_{50}(5-6 \mathrm{nM})$ value from in vitro affinity assays for the MAO-B enzyme (MAO enzymatic activity and displacement assays). PK/PD analysis per study population yielded very similar $\mathrm{E}_{\max }$ and $\mathrm{EC}_{50}$ values for patients with $\mathrm{AD}$ and $\mathrm{EC}$ subjects. Slightly lower mean $\mathrm{EC}_{50}$ values for the different ROIs were estimated for patients with $\mathrm{AD}$ compared with EC subjects. However, this analysis is limited by the small sample size, and this finding might be explained by the variability in PK and inhibition data. These data provide a good understanding of the concentration-enzyme inhibition relationship and assist in dose selection for clinical efficacy studies. Regardless of the population, sembragiline concentrations around $20 \mathrm{ng} / \mathrm{mL}$ might be needed for near-complete MAO-B inhibition in the brain.

In conclusion, sembragiline treatment was well tolerated and resulted in near-complete inhibition of the brain MAO$B$ enzyme at $1 \mathrm{mg}$ and $5 \mathrm{mg}$ daily doses in patients with $\mathrm{AD}$ and EC subjects. At these doses, near-maximal inhibition of brain MAO-B was achieved regardless of the population. In the phase 2 trial, sembragiline at 1 and $5 \mathrm{mg}$ daily failed on the primary efficacy outcome on cognition (Alzheimer's Disease Assessment Scale-cognitive subscale (ADAS-Cog11) but showed a trend toward an effect on functioning and potential for an effect on neuropsychiatric symptoms compared with placebo. The lack of a cognitive effect in moderate $\mathrm{AD}$ is consistent with the effect of the MAO-B inhibitor, selegiline, which showed no long-term benefit on cognition in patients with moderate-to-severe $\mathrm{AD}$ [11]; rather, a treatment effect has been observed only in 4-6- or 8-17-week studies [27]. The effects of sembragiline are also not in accord with the results on cognition and functioning observed with the MAO-B inhibitor lazabemide. Together, these studies suggest that MAO-B inhibitors administered in addition to background therapy (i.e. acetylcholinesterase inhibitors, memantine) in patients with moderate AD may not add to the cognitive benefit.

Acknowledgments The authors would like to thank all members of the Karolinska Insitutet PET Centre for assistance with the PET experiments. Support for third-party editorial assistance for this manuscript, furnished by Judy Wang, PhD (MediTech Media), was provided by F. HoffmannLa Roche.

Compliance with ethical standards All procedures involving human participants were in accordance with the ethical standards of the institutional and/or national research committee and with the 1964 Declaration of Helsinki and its later amendments or comparable ethical standards, whichever afforded the greater protection to the individual.

Funding and disclosures This study was sponsored by F. HoffmannLa Roche Ltd. At the time of the study, Stefan Sturm, Stephane Nave, Candice Jamois and Benedicte Ricci were employees of F. Hoffmann-La Roche. Nicholas Seneca, Robert Comley and Zbigniew Ejduk were employees of Roche at the time of this study and are currently employees of AstraZeneca Translational Science Center, AbbVie Inc. and Miedzyleski 
Specialistic Hospital, respectively. The authors have no other conflicts of interest to disclose.

Open Access This article is distributed under the terms of the Creative Commons Attribution 4.0 International License (http:// creativecommons.org/licenses/by/4.0/), which permits unrestricted use, distribution, and reproduction in any medium, provided you give appropriate credit to the original author(s) and the source, provide a link to the Creative Commons license, and indicate if changes were made.

\section{References}

1. Saura J, Andres N, Andrade C, Ojuel J, Eriksson K, Mahy N. Biphasic and region-specific MAO-B response to aging in normal human brain. Neurobiol Aging. 1997;18(5):497-507.

2. Fowler JS, Volkow ND, Wang GJ, Logan J, Pappas N, Shea C, et al. Age-related increases in brain monoamine oxidase B in living healthy human subjects. Neurobiol Aging. 1997;18(4):431-5.

3. Reinikainen KJ, Paljarvi L, Halonen T, Malminen O, Kosma VM, Laakso M, et al. Dopaminergic system and monoamine oxidase-B activity in Alzheimer's disease. Neurobiol Aging. 1988;9(3): 245-52.

4. Strolin Benedetti M, Dostert P. Monoamine oxidase, brain ageing and degenerative diseases. Biochem Pharmacol. 1989;38(4): 555-61.

5. Nakamura S, Kawamata T, Akiguchi I, Kameyama M, Nakamura N, Kimura H. Expression of monoamine oxidase B activity in astrocytes of senile plaques. Acta Neuropathol. 1990;80(4):419-25.

6. Saura J, Luque JM, Cesura AM, Da Prada M, Chan-Palay V, Huber $\mathrm{G}$, et al. Increased monoamine oxidase B activity in plaqueassociated astrocytes of Alzheimer brains revealed by quantitative enzyme radioautography. Neuroscience. 1994;62(1):15-30.

7. Kennedy BP, Ziegler MG, Alford M, Hansen LA, Thal LJ, Masliah E. Early and persistent alterations in prefrontal cortex MAO A and B in Alzheimer's disease. J Neural Transm. 2003;110(7):789-801.

8. Riederer P, Danielczyk W, Grunblatt E. Monoamine oxidase-B inhibition in Alzheimer's disease. Neurotoxicology. 2004;25(1-2): 271-7.

9. Roth BL, Hanizavareh SM, Blum AE. Serotonin receptors represent highly favorable molecular targets for cognitive enhancement in schizophrenia and other disorders. Psychopharmacology (Berl). 2004;174(1):17-24.

10. Mitchell RA, Herrmann N, Lanctot KL. The role of dopamine in symptoms and treatment of apathy in Alzheimer's disease. CNS Neurosci Ther. 2011;17(5):411-27.

11. Sano M, Ernesto C, Thomas RG, Klauber MR, Schafer K, Grundman M, et al. A controlled trial of selegiline, alpha-tocopherol, or both as treatment for Alzheimer's disease. The Alzheimer's Disease Cooperative Study. N Engl J Med. 1997;336(17):1216-22.

12. Magni G, Meibach RC. Lazabemide for the long-term treatment of Alzheimer's disease. Eur Neuropsychopharmacol. 1999;9((Supplement 5)):142.

13. Borroni E, Wyler R, Messer J, Nave S, Cesura A. Preclinical characterization of R04602522, a novel, selective, and orally active monoamine oxidase type B Inhibitor for the treatment of Alzheimer's Disease. Alzheimers Dement. 2013;9:818.

14. Berlin I, Hunneyball IM, Greiling D, Jones SP, Fuder H, Stahl HD. A selective reversible monoamine oxidase $\mathrm{B}$ inhibitor in smoking cessation: effects on its own and in association with transdermal nicotine patch. Psychopharmacology (Berl). 2012;223(1):89-98.

15. Nagren K, Muller L, Halldin C, Swahn CG, Lehikoinen P. Improved synthesis of some commonly used PET radioligands by the use of [11C]methyl triflate. Nucl Med Biol. 1995;22(2): 235-9.

16. Tzourio-Mazoyer N, Landeau B, Papathanassiou D, Crivello F, Etard O, Delcroix N, et al. Automated anatomical labeling of activations in SPM using a macroscopic anatomical parcellation of the MNI MRI single-subject brain. Neuroimage. 2002;15(1):273-89.

17. Wienhard K, Dahlbom M, Eriksson L, Michel C, Bruckbauer T, Pietrzyk U, et al. The ECAT EXACT HR: performance of a new high resolution positron scanner. J Comput Assist Tomogr. 1994;18(1):110-8.

18. Bench CJ, Price GW, Lammertsma AA, Cremer JC, Luthra SK, Turton D, et al. Measurement of human cerebral monoamine oxidase type B (MAO-B) activity with positron emission tomography (PET): a dose ranging study with the reversible inhibitor Ro 196327. Eur J Clin Pharmacol. 1991;40(2):169-73.

19. Fowler JS, Wang GJ, Logan J, Xie S, Volkow ND, MacGregor RR, et al. Selective reduction of radiotracer trapping by deuterium substitution: comparison of carbon-11-L-deprenyl and carbon-11deprenyl-D2 for MAO B mapping. J Nucl Med. 1995;36(7): 1255-62.

20. Logan J, Fowler JS, Volkow ND, Wang GJ, MacGregor RR, Shea C. Reproducibility of repeated measures of deuterium substituted $[11 \mathrm{C}] \mathrm{L}$-deprenyl ([11C]L-deprenyl-D2) binding in the human brain. Nucl Med Biol. 2000;27(1):43-9.

21. Nave S, Doody RS, Boada M, Grimmer T, Savola J, Delmar P, et al. Sembragiline in moderate Alzheimer's disease dementia: results of a phase 2 trial (MAyflOwer RoAD). J Prevent Alzheimer's Dis. 2015;2:276.

22. Fowler JS, Volkow ND, Logan J, Wang GJ, MacGregor RR, Schyler D, et al. Slow recovery of human brain MAO B after Ldeprenyl (Selegeline) withdrawal. Synapse. 1994;18(2):86-93.

23. Hirvonen J, Kailajarvi M, Haltia T, Koskimies S, Nagren K, Virsu P, et al. Assessment of MAO-B occupancy in the brain with PET and [11C]-L-deprenyl-D2: a dose-finding study with a novel MAO-B inhibitor, EVT 301. Clin Pharmacol Ther. 2009;85(5):506-12.

24. Carter SF, Scholl M, Almkvist O, Wall A, Engler H, Langstrom B, et al. Evidence for astrocytosis in prodromal Alzheimer disease provided by $11 \mathrm{C}$-deuterium-L-deprenyl: a multitracer PET paradigm combining 11C-Pittsburgh compound B and 18F-FDG. J Nucl Med. 2012;53(1):37-46.

25. Fowler JS, MacGregor RR, Wolf AP, Arnett CD, Dewey SL, Schlyer D, et al. Mapping human brain monoamine oxidase A and B with 11C-labeled suicide inactivators and PET. Science. 1987;235(4787):481-5.

26. Fowler JS, Logan J, Wang GJ, Volkow ND. Monoamine oxidase and cigarette smoking. Neurotoxicology. 2003;24(1):75-82.

27. Birks J, Flicker L. Selegiline for Alzheimer's disease. Cochrane Database Syst Rev. 2003;(1)(1):CD000442. 